\title{
Fabrication of low-loss silicon-on-oxidized-porous- silicon strip waveguide using focused proton-beam irradiation
}

\author{
E. J. Teo, ${ }^{1, *}$ A. A. Bettiol, ${ }^{1}$ P. Yang, ${ }^{2}$ M. B. H. Breese, ${ }^{1}$ B. Q. Xiong, ${ }^{1}$ G. Z. Mashanovich, ${ }^{2}$ \\ W. R. Headley, ${ }^{2}$ and G. T. Reed $^{2}$ \\ ${ }^{1}$ Centre for Ion Beam Applications, Department of Physics, National University of Singapore, \\ 2 Science Drive 3, Singapore 117542 \\ ${ }^{2}$ Advanced Technology Institute, University of Surrey, GU2 7XH, United Kingdom \\ *Corresponding author: phytej@nus.edu.sg
}

Received December 10, 2008; accepted December 20, 2008;

posted January 29, 2009 (Doc. ID 105122); published February 25, 2009

\begin{abstract}
We have successfully fabricated low-loss silicon-on-oxidized-porous-silicon (SOPS) strip waveguides with high-index contrast using focused proton-beam irradiation and electrochemical etching. Smooth surface quality with rms roughness of $3.1 \mathrm{~nm}$ is achieved for a fluence of $1 \times 10^{15} / \mathrm{cm}^{2}$ after postoxidation treatment. Optical characterization at a wavelength of $1550 \mathrm{~nm}$ shows a loss of $1.1 \pm 0.4 \mathrm{~dB} / \mathrm{cm}$ and $1.2 \pm 0.4 \mathrm{~dB} / \mathrm{cm} \mathrm{in}$ TE and TM polarization respectively, which we believe is the lowest reported loss for SOPS waveguides. This opens up new opportunities for all-silicon-based optoelectronics applications. (c) 2009 Optical Society of America

OCIS codes: $230.7370,220.4610,290.5880$.
\end{abstract}

Porous silicon (PS) is a material that offers many advantages for optical-waveguide applications in allsilicon optoelectronics integrated devices. PS is easy to implement and has a widely adjustable refractive index from 1.2 to 3 that can be varied in depth with an arbitrary profile by changing the current density [1]. Waveguides can be grown to produce a low-highlow refractive index profile. This approach has already been used to form multilayer planar waveguides [2,3], strip-rib waveguides [3], or buried waveguides [4]. However, a relatively high loss of $5-10 \mathrm{~dB} / \mathrm{cm}$ is obtained for these waveguides owing to absorption and interface scattering [2-4]. Oxidation can be used to improve the loss, but this reduces the refractive index contrast between the core and cladding and limits its use for applications that require tight confinement of light such as integrated photonics circuits.

In this Letter, we demonstrate the ability to fabricate high-index-contrast silicon-on-oxidized-PS (SOPS) strip waveguide. This process is compatible with full isolation by oxidized porous silicon (FIPOS) [5]. A finely focused beam of protons is used to create localized lines of high resistivity in silicon, which is prevented from etching during subsequent anodization. The resultant structure consists of a silicon core that is optically isolated by the PS cladding. In terms of refractive index contrast and structure profile, the SOPS waveguide is similar to a conventional siliconon-insulator (SOI) waveguide, with the added advantages that it can be produced directly in silicon without the need for an SOI substrate, and fewer complex processing steps are required.

Owing to the high refractive index difference between the oxidized PS and silicon $(\Delta n \sim 2)$, surface roughness contributes a significant factor to the propagation loss. Previously, we obtained a relatively high loss of $6.6 \mathrm{~dB} / \mathrm{cm}$ in a buried silicon waveguide with PS cladding [6]. Here, we show improved propa- gation loss of SOPS waveguides by reducing the surface roughness through optimization of the irradiation fluence and a postoxidation process.

Direct proton-beam irradiation is an emerging modification technique for fabricating waveguides in silicon and glass [6-9]. Figure 1 shows a schematic of the fabrication process, carried out using $250 \mathrm{keV}$ protons of $\sim 200 \mathrm{~nm}$ spatial resolution. We have irradiated three waveguides with fluences of $7 \times 10^{13}, 1$ $\times 10^{14}$, and $1 \times 10^{15} / \mathrm{cm}^{2}$ into a $p$-type silicon wafer $(0.5 \Omega \mathrm{cm})$ by translating an inchworm stage over a length of $1 \mathrm{~cm}$. To create the SOPS strip structure, the sample is electrochemically etched in a two-step process. First, it is etched in $12 \%$ HF with a current density of $100 \mathrm{~mA} / \mathrm{cm}^{2}$ for $74 \mathrm{~s}$. The overlying PS is removed using dilute potassium hydroxide. A second etching process is performed using $24 \% \mathrm{HF}$ with a current density of $40 \mathrm{~mA} / \mathrm{cm}^{2}$ for $100 \mathrm{~s}$ to provide the bottom's cladding. Oxidation is then used to simultaneously anneal out the proton-induced damage and to reduce the surface roughness. First, the sample is annealed in air ambient at $300^{\circ} \mathrm{C}$ for $3 \mathrm{~h}$ to stabilize the PS structure, followed by $1000^{\circ} \mathrm{C}$ for $3 \mathrm{~h}$ to fully
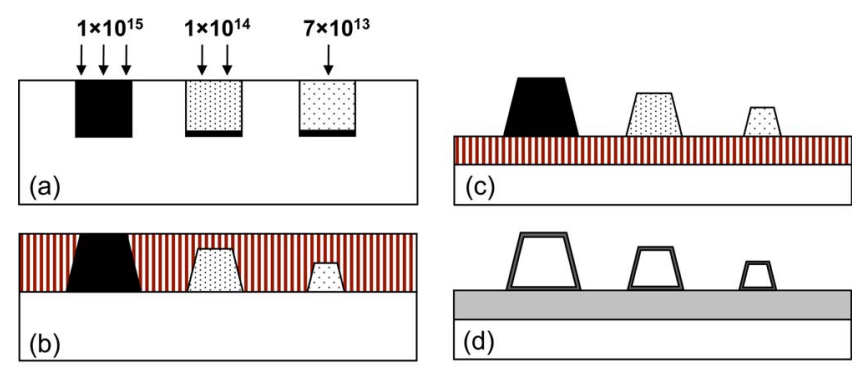

III PS $\square$ Si oxide

$\square$ oxidized PS $\square$ silicon

Fig. 1. (Color online) Schematics of the fabrication process showing (a) direct proton-beam irradiation, (b) PS formation till the end of ion range, (c) a second etching step to undercut the irradiated structures, and (d) the oxidation step. 
oxidize the PS layer [10].

Figure 2 shows the cross-sectional scanningelectron microscopy (SEM) images of three waveguides irradiated with a lateral scan size of $2 \mu \mathrm{m}$. The resultant structures have a trapezoidal core, in which the cross-sectional area depends on the fluence. Postoxidation produces a $180 \mathrm{~nm}$ layer of oxide around the core circumference. By increasing the fluence from $7 \times 10^{13} / \mathrm{cm}^{2}$ to $1 \times 10^{15} / \mathrm{cm}^{2}$, the rate of PS formation slows down, reducing the PS layer thickness formed above the waveguide from $0.7 \mu \mathrm{m}$ to almost zero. This results in an increase in the core height from $1.9 \mu \mathrm{m}$ to $2.5 \mu \mathrm{m}$ and width from $1.7 \mu \mathrm{m}$ to $2.9 \mu \mathrm{m}$. At $1 \times 10^{15} / \mathrm{cm}^{2}$, no etching occurs through the irradiated regions, and this height corresponds to the proton range. The refractive index of the oxidized PS is determined to be 1.35 from fitting the reflectance spectrum using the Bruggerman model [11], producing a large refractive index difference of 2.19 between the core and cladding.

In conventional SOI waveguides, the sidewall roughness caused by the lithographic process is the main contribution to scattering loss [12]. For SOPS waveguides, the etching process induces surface roughness on all sides, which is detrimental to scattering loss. Atomic force microscopy (AFM) is used to characterize the rms roughness $\sigma$ for each fluence, before and after oxidation. Owing to the rounded edges of the waveguides, we have taken an average $\sigma$ across the top and sidewall surfaces, from an AFM image size of $5 \times 5 \mu \mathrm{m}$. This is plotted as a function of height of the structure shown in Fig. 3. The error bars show the variation of $\sigma$ measured over five different sections of each waveguide.

Before oxidation, the unirradiated surface at the PS/Si interface shows a $\sigma$ of $17.5 \mathrm{~nm}$. As the waveguide height increases from $1.9 \mu \mathrm{m}$ to $2.5 \mu \mathrm{m}$ with increasing fluence, $\sigma$ decreases from $10.0 \mathrm{~nm}$ to $6.5 \mathrm{~nm}$. Fluctuations at the PS dissolution front arise from the local variation of the current density of the initial silicon surface [13]. In the irradiated regions, the surface roughness is determined by the uniformity of the beam irradiation. The higher surface roughness for low fluence is due to the increase in sensitivity to beam fluctuation, since the structure height changes rapidly in this fluence range [6]. This can be minimized by scanning many times over the waveguide structure and stabilizing the beam intensity produced from the accelerator.

To measure the $\sigma$ at the PS/Si interface after oxidation, the waveguides were dipped into dilute $\mathrm{HF}$ for a few minutes to remove the oxide. A reduction of $\sigma$ by about 3-4 $\mathrm{nm}$ was observed in the irradiated regions and $6 \mathrm{~nm}$ in the unirradiated region. A mini-

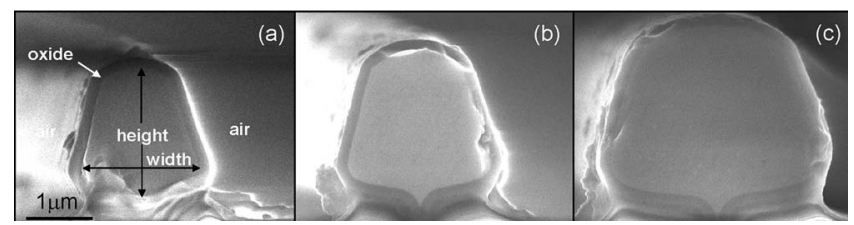

Fig. 2. SEM of the waveguides irradiated with fluences of (a) $7 \times 10^{13}$, (b) $1 \times 10^{14}$, and (c) $1 \times 10^{15} / \mathrm{cm}^{2}$.

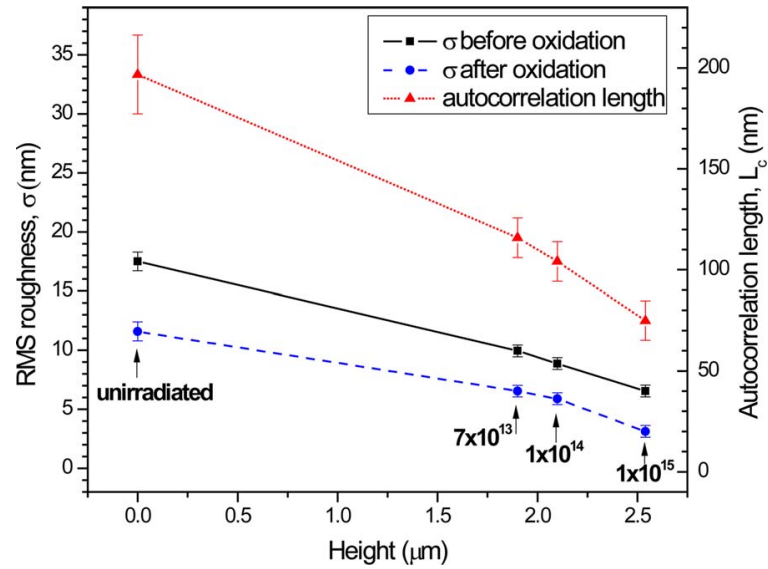

Fig. 3. (Color online) RMS roughness $(\sigma)$ and autocorrelation length $\left(L_{c}\right)$ of the three waveguides as a function of height.

mum $\sigma$ of $3.1 \mathrm{~nm}$ was obtained with the highest fluence at the waveguide height of $2.5 \mu \mathrm{m}$. Oxidation occurs faster at the convex of the rough $\mathrm{Si}$, leading to a smoother surface. This result is comparable to $\sigma$ of about $10 \mathrm{~nm}$ and $2 \mathrm{~nm}$ obtained before and after oxidation for SOI strip waveguide fabricated using state-of-the-art lithography and etching techniques [14]. Besides surface roughness, autocorrelation length $L_{c}$ also affects the scattering loss [12]. We observed that the unirradiated PS/Si interface shows an $L_{c}$ of $200 \mathrm{~nm}$, which reduces from $117 \mathrm{~nm}$ to $75 \mathrm{~nm}$ with increasing height and fluence.

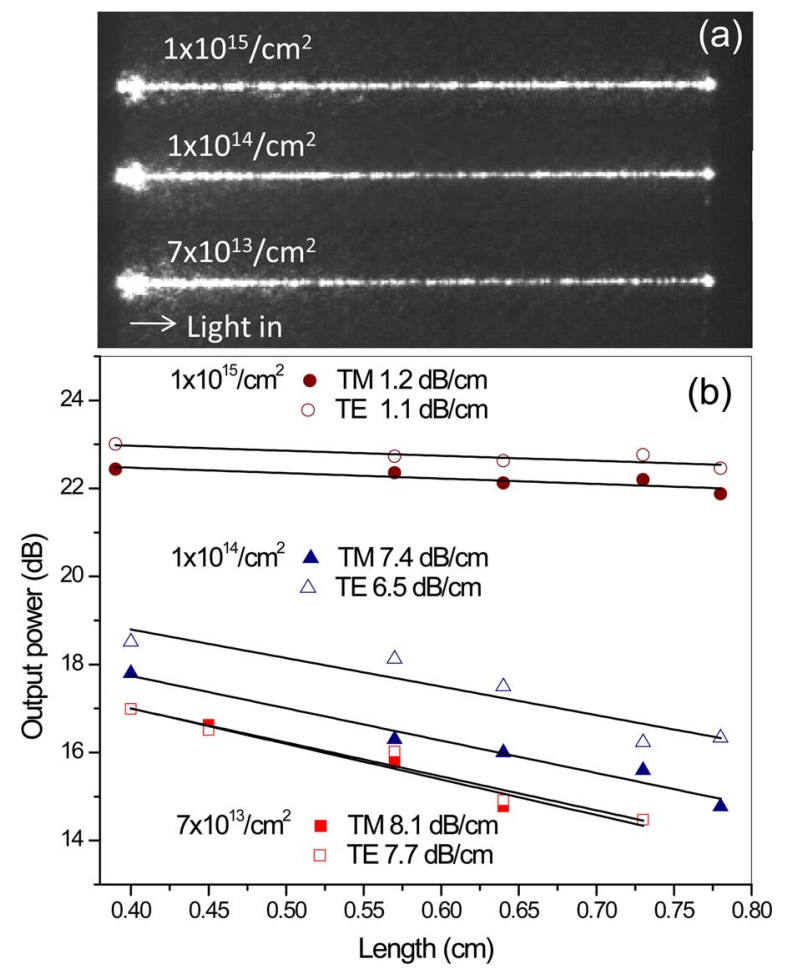

Fig. 4. (Color online) (a) Scattered light from the three waveguides, (b) loss data for $7 \times 10^{13} / \mathrm{cm}^{2}$ ( $\square$, 口), 1 $\times 10^{14} / \mathrm{cm}^{2}(\triangle, \mathbf{\Delta})$, and $1 \times 10^{15} / \mathrm{cm}^{2}(\bigcirc, \bullet)$ after oxidation. The empty shapes represent TE polarization, and the filled shapes represent TM polarization. The straight line shows a linear fit of the output power $(\mathrm{dB})$ as a function of length. 
Table 1. Waveguide Dimensions, $\sigma, L_{c}$, and Measured Losses for Each Waveguide Fluence after Oxidation

\begin{tabular}{ccccc}
\hline $\begin{array}{c}\text { Fluence } \\
\left(/ \mathrm{cm}^{2}\right)\end{array}$ & $\begin{array}{c}\text { Width } \\
\text { Height } \\
\left(\mu \mathrm{m}^{2}\right)\end{array}$ & $\begin{array}{c}\sigma \\
(\mathrm{nm})\end{array}$ & $\begin{array}{c}L_{c} \\
(\mathrm{~nm})\end{array}$ & $\begin{array}{c}\text { Loss TE } \\
(\mathrm{dB} / \mathrm{cm})\end{array}$ \\
\hline $7 \times 10^{13}$ & $1.7 \times 1.9$ & 6.5 & 117 & $7.7 \pm 0.8$ \\
$1 \times 10^{14}$ & $2.1 \times 2.1$ & 5.9 & 105 & $6.5 \pm 0.8$ \\
$1 \times 10^{15}$ & $2.9 \times 2.5$ & 3.1 & 75 & $1.1 \pm 0.4$ \\
\hline
\end{tabular}

Optical characterization was carried out in both TE and TM polarizations at $1550 \mathrm{~nm}$. A tunable diode laser is coupled into the waveguide using a $60 \times$ objective lens. A polarizing beam splitter and a halfwave plate were inserted into the beam path, enabling discrimination between the TE and TM polarizations. The scattered light from the top of the waveguides was monitored using a highly sensitive InGaAs IR camera (Xeva-FPA-1.7-320), and the output light is imaged using an IR Vidicon camera (Electrophysics 7290A).

Figure 4(a) shows the light scattered off the top of the waveguides over a length of $0.73 \mathrm{~cm}$. Propagation loss was measured using the cutback technique [see Fig. 4(b)]. Table 1 shows the measured TE and TM losses, $\sigma$ and $L_{c}$, for each waveguide after oxidation. As the fluence increases from $7 \times 10^{13} / \mathrm{cm}^{2}$ to 1 $\times 10^{15} / \mathrm{cm}^{2}$ there is a significant reduction in loss, from $7.7 \mathrm{~dB} / \mathrm{cm}$ to $1.1 \mathrm{~dB} / \mathrm{cm}$ for TE polarization and $8.1 \mathrm{~dB} / \mathrm{cm}$ to $1.2 \mathrm{~dB} / \mathrm{cm}$ for TM polarization. Scattering loss of conventional SOI strip waveguide shows a $\sigma^{2}$ dependence on surface roughness [12]. A stronger surface-roughness effect is expected for SOPS waveguides owing to an additional contribution to the scattering loss from the top and bottom surfaces. It is shown here that by reducing the surface roughness by a factor of two, the propagation loss is improved significantly. Here, the influence of core size change from 2 to $3 \mu \mathrm{m}$ on propagation loss is relatively small [14]. Currently our losses are much better than $5-10 \mathrm{~dB} / \mathrm{cm}$ reported for PS waveguides $[3,4]$.

In conclusion, low-loss SOPS waveguide with a high refractive index was successfully fabricated using direct proton-beam irradiation. The surface roughness caused by the etching process is the main contribution to the propagation loss and can be reduced to $3.1 \mathrm{~nm}$ for a fluence of $1 \times 10^{15} / \mathrm{cm}^{2}$ with postoxidation. Under such conditions, a propagation loss of approximately $1 \mathrm{~dB} / \mathrm{cm}$ for both the $\mathrm{TE}$ and TM polarizations can be obtained, which to our knowledge is the lowest reported loss for SOPS waveguides. This process can be extended to existing FIPOS technology for all-silicon-based integrated photonics applications.

E. J. Teo acknowledges financial support from Lee Kuan Yew Fellowship funded by Minstry of Education-Academic Research Fund.

\section{References}

1. L. Pavesi, Riv. Nuovo Cimento 20, 1 (1997).

2. P. Ferrand and R. Romestain, Appl. Phys. Lett. 77, 3535 (2000).

3. A. Loni, L. T. Canham, M. G. Berger, R. Arens-Fischer, H. Munderm, H. Luth, H. F. Arrand, and T. M. Benson, Thin Solid Films 276, 143 (1996).

4. M. Takahashi and N. Koshida, J. Appl. Phys. 86, 5274 (1999).

5. K. Imai, Solid-State Electron. 24, 150 (1981).

6. E. J. Teo, A. A. Bettiol, M. B. H. Breese, P. Yang, G. Z. Mashanovich, W. R. Headley, G. T. Reed, and D. J. Blackwood, Opt. Express 16, 573 (2008).

7. E. J. Teo, M. B. H Breese, E. P. Tavernier, A. A. Bettiol, F. Watt, M. H. Liu, and D. J. Blackwood, Appl. Phys. Lett. 84, 3202 (2004).

8. A. A. Bettiol, S. V. Rao, E. J. Teo, J. A. Van Kan, and F. Watt, Appl. Phys. Lett. 88, 171106 (2006).

9. P. Yang, G. Mashanovich, I. Gomez-Morilla, W. R. Headley, G. T. Reed, E. J. Teo, D. J. Blackwood, M. B. H. Breese, and A. A. Bettiol, Appl. Phys. Lett. 90, 241109 (2007).

10. T. L. Lin and K. L. Wang, Appl. Phys. Lett. 49, 1104 (1986).

11. D. E. Aspnes, Thin Solid Films 89, 249 (1982).

12. K. K. Lee, D. R. Lim, H. C. Luan, A. Agarwal, J. Foresi, and L. C. Kimerling, Appl. Phys. Lett. 77, 1617 (2000).

13. G. Lerondel, R. Romestain, and S. Barret, J. Appl. Phys. 81, 6171 (1997).

14. K. K. Lee, D. R. Lim, L. C. Kimerling, J. Shin, and F. Cerrina, Opt. Lett. 26, 1888 (2001). 